\title{
CP symmetry tests in the cascade-anticascade decay of charmonium
}

\author{
Patrik Adlarson $\odot^{1, *}$ and Andrzej Kupsc ${ }^{1,2, \dagger}$ \\ ${ }^{1}$ Department of Physics and Astronomy, Uppsala University, Box 516, SE-75120 Uppsala, Sweden \\ ${ }^{2}$ National Centre for Nuclear Research, Pasteura 7, 02-093 Warsaw, Poland
}

(Received 6 September 2019; published 3 December 2019)

\begin{abstract}
We analyze joint angular distributions of a charmonium decay to the $\Xi \bar{\Xi}$ pair using the $\Xi \rightarrow \Lambda \pi \rightarrow p \pi^{-} \pi$ weak decay chain for the cascade and the charge conjugated mode for the anticascade. The decays allow a direct comparison of the baryon and antibaryon decay properties and a sensitive test of $C P$ symmetry in the strange baryon sector. We show that all involved decay parameters can be determined separately in vector and (pseudo)scalar charmonia decays into $\Xi \bar{\Xi}$ due to the spin correlations between the weak decay chains. Contrary to the recently measured $e^{+} e^{-} \rightarrow J / \psi \rightarrow \Lambda \bar{\Lambda}$ process, the transverse polarization of the cascade is not needed and has almost no impact on the uncertainties of the decay parameters.
\end{abstract}

DOI: $10.1103 /$ PhysRevD.100.114005

\section{INTRODUCTION}

The ongoing experimental studies of the combined charge conjugation parity $(C P)$ symmetry violation in particle decays aim to find effects that are not expected in the Standard Model (SM), such that new dynamics is revealed. The existence of $C P$ violation in kaon and beauty meson decays is well established [1-3]. The first observation of the $C P$ violation for charm mesons was reported this year by the $\mathrm{LHCb}$ experiment [4] and in the bottom baryon sector evidence is mounting [5]. All the observations are consistent with the SM expectation. However, no signal is detected in decays of baryons with strange quark(s) (hyperons). Hyperon decays offer promising possibilities for such searches as they are sensitive to sources of $C P$ violation that neutral kaon decays are not [6]. A signal of $C P$ violation can be a difference in decay distributions between the charge conjugated decay modes. The main decay modes of the ground state hyperons are weak transitions into a baryon and a pseudoscalar meson like $\Lambda \rightarrow p \pi^{-}$, branching fraction $\mathcal{B} \approx 64 \%$, and $\Xi^{-} \rightarrow \Lambda \pi^{-}, \mathcal{B} \approx 100 \%$ [7]. They involve two amplitudes: parity conserving to the relative $p$ state, and parity violating to the $s$ state. The angular distribution and the polarization of the daughter baryon are described by two decay parameters: the decay asymmetry $\alpha=2 \operatorname{Re}\left(s^{*} p\right) /\left(|p|^{2}+|s|^{2}\right)$ and the relative phase $\phi=\arg (s / p)$. Here, we denote decay asymmetries for

\footnotetext{
*Patrik.Adlarson@physics.uu.se

†Andrzej.Kupsc@physics.uu.se
}

Published by the American Physical Society under the terms of the Creative Commons Attribution 4.0 International license. Further distribution of this work must maintain attribution to the author(s) and the published article's title, journal citation, and DOI. Funded by SCOAP.
$\Lambda \rightarrow p \pi^{-}$and $\Xi^{-} \rightarrow \Lambda \pi^{-}$as $\alpha_{\Lambda}$ and $\alpha_{\Xi}$, respectively. In the $C P$ symmetry conserving limit the parameters $\alpha$ and $\phi$ for the charge conjugated decay mode have the same absolute values but opposite signs, e.g., $\alpha_{\Lambda}=-\alpha_{\bar{\Lambda}}$. The best limit for $C P$ violation in the strange baryon sector was obtained by comparing the $\Xi^{-}$and $\bar{\Xi}^{+}$decay chains of unpolarized $\Xi$ baryons at the HyperCP (E871) experiment [8] by determining the asymmetry $A_{\Xi \Lambda}=\left(\alpha_{\Lambda} \alpha_{\Xi}-\alpha_{\bar{\Lambda}} \alpha_{\bar{\Xi}}\right) /$ $\left(\alpha_{\Lambda} \alpha_{\Xi}+\alpha_{\bar{\Lambda}} \alpha_{\Xi}\right)$. The result, $A_{\Xi \Lambda}=(0.0 \pm 5.1 \pm 4.7) \times$ $10^{-4}$, is consistent with the SM predictions: $\left|A_{\Xi \Lambda}\right| \leq 5 \times$ $10^{-5}$ [9]. However, a preliminary HyperCP result presented at the BEACH 2008 Conference suggests a large value of the asymmetry $A_{\Xi \Lambda}=(-6.0 \pm 2.1 \pm 2.0) \times 10^{-4}[10]$.

With a well-defined initial state charmonium decay into a strange baryon-antibaryon pair offers an ideal system to test fundamental symmetries. Vector charmonia $J / \psi$ and $\psi^{\prime}$ can be directly produced in an electron-positron collider with large yields and have relatively large branching fractions into a hyperon-antihyperon pair, see Table I. With the world's largest sample of $10^{10} \mathrm{~J} / \psi$ collected at BESIII $[11,12]$ detailed studies of the hyperon-antihyperon systems are possible. The potential impact of such measurements was shown in the recent analysis using a data set of $4.2 \times 10^{5} e^{+} e^{-} \rightarrow J / \psi \rightarrow \Lambda \bar{\Lambda}$ events reconstructed via $\Lambda \rightarrow p \pi^{-}+$c.c. decay chain and has lead, e.g., to the major revision of the $\alpha_{\Lambda}$ value [13]. The determination of the asymmetry parameters was possible only due to the transverse polarization and the spin correlations of the $\Lambda$ and $\bar{\Lambda}$. In the analysis the complete multidimensional information of the final state particles was used in an unbinned maximum $\log$ likelihood fit to the fully differential angular expressions from Ref. [14]. The method allows for a direct comparison of the decay parameters of the charge conjugate decay modes and a test of the $C P$ symmetry. 
TABLE I. Branching fractions for some $J / \psi, \psi^{\prime} \rightarrow B \bar{B}$ decays and the estimated sizes of the data samples from the full data set of $10^{10} \mathrm{~J} / \psi$ and $3.2 \times 10^{9} \psi^{\prime}$ in the BESIII proposal [11]. The approximate detection efficiencies for the final states reconstructed using $\Lambda \rightarrow p \pi^{-}$and $\Xi \rightarrow \Lambda \pi$ decay modes are based on the published BESIII analyses using partial data sets [15-17].

\begin{tabular}{lcccc}
\hline \hline Decay mode & $\mathcal{B}\left(\right.$ units $\left.10^{-4}\right)$ & Angular distribution parameter $\alpha_{\psi}$ & Detection efficiency & No. events expected at BESIII \\
\hline$J / \psi \rightarrow \Lambda \bar{\Lambda}$ & $19.43 \pm 0.03 \pm 0.33$ & $0.469 \pm 0.026$ & $40 \%$ & $3200 \times 10^{3}$ \\
$\psi(2 S) \rightarrow \Lambda \bar{\Lambda}$ & $3.97 \pm 0.02 \pm 0.12$ & $0.824 \pm 0.074$ & $40 \%$ & $650 \times 10^{3}$ \\
$J / \psi \rightarrow \Xi^{0} \bar{\Xi}^{0}$ & $11.65 \pm 0.04$ & $0.66 \pm 0.03$ & $14 \%$ & $670 \times 10^{3}$ \\
$\psi(2 S) \rightarrow \Xi^{0} \bar{\Xi}^{0}$ & $2.73 \pm 0.03$ & $0.65 \pm 0.09$ & $14 \%$ & $160 \times 10^{3}$ \\
$J / \psi \rightarrow \Xi^{-} \bar{\Xi}^{+}$ & $10.40 \pm 0.06$ & $0.58 \pm 0.04$ & $19 \%$ & $810 \times 10^{3}$ \\
$\psi(2 S) \rightarrow \Xi^{-} \bar{\Xi}^{+}$ & $2.78 \pm 0.05$ & $0.91 \pm 0.13$ & $19 \%$ & $210 \times 10^{3}$ \\
\hline \hline
\end{tabular}

In Ref. [18] we have extended the formalism to describe processes which include decay chains of multistrange hyperons like the $e^{+} e^{-} \rightarrow \Xi \bar{\Xi}$ reaction with the $\Xi \rightarrow \Lambda \pi, \Lambda \rightarrow$ $p \pi^{-}+$c.c. decay sequences. The expressions are much more complicated than the single step weak decays in $e^{+} e^{-} \rightarrow \Lambda \bar{\Lambda}$. In this paper we use the joint distributions for $e^{+} e^{-} \rightarrow \Xi \bar{\Xi}$ to show that the role of the transverse polarization is fully replaced by the diagonal spin correlations between the cascades. All decay parameters can be determined simultaneously and the statistical uncertainties are nearly independent on the size of the transverse polarization in the production process. In particular we find that the uncertainty for the $\alpha_{\Lambda}$ asymmetry is more than two times better than in $e^{+} e^{-} \rightarrow J / \psi \rightarrow \Lambda \bar{\Lambda}$ process for the same number of reconstructed events. A corresponding analysis of a single $\Xi(\bar{\Xi})$ baryon decay chain would require a known, non-zero initial polarization. We estimate uncertainties of the various possible $C P$ odd asymmetries which can be extracted from the exclusive analysis. We show that the same information can be extracted from an exclusive analysis of the cascade-anticascade decay of a (pseudo)scalar charmonium. Our result provides an important input to the plans for two Super Tau Charm Factories (STCF) in Novosibirsk (Russia) [19] and in Hefei (China) [20] promising data samples of more than $10^{12} \mathrm{~J} / \psi$ events, where such asymmetries can be measured with the precision close to the SM predictions.

We first summarize the formalism describing the joint angular distributions and present a method using properties of the exact likelihood function to analyze the multidimensional distributions and correlations between the decay parameters.

\section{FORMALISM}

In general, a quantum state of a baryon-antibaryon pair $B \bar{B}$ (with spin one-half) can be represented by the following spin density matrix:

$$
\sum_{\mu, \nu=0}^{3} C_{\mu \nu} \sigma_{\mu}^{B} \otimes \sigma_{\nu}^{\bar{B}},
$$

where a set of four Pauli matrices $\sigma_{\mu}^{B}\left(\sigma_{\nu}^{\bar{B}}\right)$ in the rest frame of a baryon $B(\bar{B})$ is used and $C_{\mu \nu}$ is $4 \times 4$ real matrix representing polarizations and spin correlations for the baryons.

Consider the $e^{+} e^{-} \rightarrow B \bar{B}$ reaction represented in Fig. 1, where the electron and positron beams are unpolarized. The spin matrices $\sigma_{\mu}^{B}$ and $\sigma_{\nu}^{\bar{B}}$ are given in the helicity frames of the baryon $B$ and antibaryon $\bar{B}$, respectively. The axes of the coordinate systems are denoted $\hat{\mathbf{x}}_{1}, \hat{\mathbf{y}}_{1}, \hat{\mathbf{z}}_{1}$ and $\hat{\mathbf{x}}_{2}, \hat{\mathbf{y}}_{2}, \hat{\mathbf{z}}_{2}$. The baryons and antibaryon can have aligned or opposite helicities. Due to the parity conservation only two transitions are independent and the $C_{\mu \nu}$ matrix can be parametrized by: $\alpha_{\psi}$-baryon angular distribution parameter, $-1 \leq \alpha_{\psi} \leq 1$, and $\Delta \Phi$-relative phase between the two transitions. The elements of the $C_{\mu \nu}$ matrix are functions of the scattering angle $\theta$ of the $B$ baryon [18]:

$$
\left(\begin{array}{cccc}
1+\alpha_{\psi} \cos ^{2} \theta & 0 & \beta_{\psi} \sin 2 \theta & 0 \\
0 & \sin ^{2} \theta & 0 & \gamma_{\psi} \sin 2 \theta \\
-\beta_{\psi} \sin 2 \theta & 0 & \alpha_{\psi} \sin ^{2} \theta & 0 \\
0 & -\gamma_{\psi} \sin 2 \theta & 0 & -\alpha_{\psi}-\cos ^{2} \theta
\end{array}\right) \text {, }
$$

where $\beta_{\psi}$ and $\gamma_{\psi}$ (real parameters) are defined as: $\gamma_{\psi}+i \beta_{\psi}=\frac{1}{2} \sqrt{1-\alpha_{\psi}^{2}} \exp (i \Delta \Phi)$. The polarization vector of $B(\bar{B})$ can have only $\hat{\mathbf{y}}_{1}\left(\hat{\mathbf{y}}_{2}\right)$ component and the value is $\beta_{\psi} \sin 2 \theta /\left(1+\alpha_{\psi} \cos ^{2} \theta\right)$, i.e., the polarization is zero if $\beta_{\psi}=0$. In the limit of large c.m. energies $\alpha_{\psi}=1$ implying $\beta_{\psi}=\gamma_{\psi}=0$ [21] and diagonal $C_{\mu \nu}$. For the $B \bar{B}$ decay of a (pseudo)scalar charmonium (like $\eta_{c}$ or $\chi_{c 0}$ ) the initial state is spin singlet and the spin orientations of the baryon and antibaryon are opposite. Therefore $C_{\mu \nu}$ is $\operatorname{diag}(1,-1,1,1)$, where the signs are stipulated by the relative orientation of the axes of the $B$ and $\bar{B}$ helicity frames shown in Fig. 1. The direction of the $\hat{\mathbf{z}}$ axis is arbitrary.

In a weak hadronic decay $D$ of a spin one-half baryon to a spin one-half baryon and a pseudoscalar meson: $B_{A} \rightarrow B_{B}+P$, the initial and final states can be represented by linear combinations of the Pauli density matrices $\sigma_{\mu}^{B_{A}}$ and $\sigma_{\nu}^{B_{B}}$, defined in the helicity frame of $B_{A}$ and $B_{B}$, respectively. It is enough to know how each base spin 


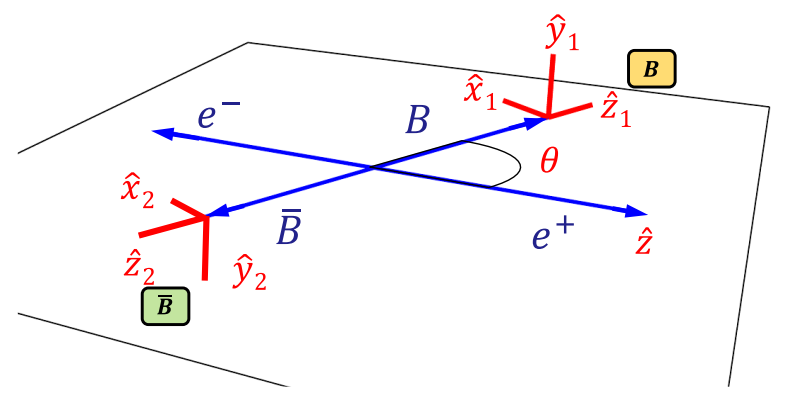

FIG. 1. Orientation of the axes in baryon $B$ and antibaryon $\bar{B}$ helicity frames.

matrix transforms under a decay process. One can therefore represent the weak decay by a decay matrix $a_{\mu \mu^{\prime}}^{D}$ which transforms the base matrices [18]:

$$
\sigma_{\mu}^{B_{A}} \rightarrow \sum_{\mu^{\prime}=0}^{3} a_{\mu \mu^{\prime}}^{D} \sigma_{\mu^{\prime}}^{B_{B}}
$$

The decay matrix depends on two decay parameters: $-1 \leq$ $\alpha_{D} \leq 1$ and $-\pi \leq \phi_{D}<\pi$ according to the Particle Data Group (PDG) convention [7]. Often, two related decay parameters $\beta_{D}$ and $\gamma_{D}$ are used, where $\beta_{D}=\sqrt{1-\alpha_{D}^{2}} \sin \phi_{D}$ and $\gamma_{D}=\sqrt{1-\alpha_{D}^{2}} \cos \phi_{D}$. The elements of the $4 \times 4$ decay matrix $a_{\mu \nu}^{D} \equiv a_{\mu \nu}\left(\theta, \varphi ; \alpha_{D}, \phi_{D}\right)$ depend on the kinematic variables $\theta$ and $\varphi$, the spherical coordinates of the $B_{B}$ momentum in the $B_{A}$ helicity frame, and on the decay parameters $\alpha_{D}$ and $\phi_{D}$. The explicit form of the $a_{\mu \nu}^{D}$ is given in Ref. [18], where a two angle helicity rotation matrix convention is used. If the polarization of the baryon $B_{B}$ is not measured the decay is described by the $a_{\mu 0}^{D}$ elements of the decay matrix and only the $\alpha_{D}$ parameter is involved. This is normally the case for $\Lambda \rightarrow p \pi^{-}$since the proton polarization determination would require a dedicated detection system. A complete joint angular distribution of a hyperon-antihyperon pair production process including the weak decay chains is obtained by the application of Eq. (1), the decay matrices transformations Eq. (3) and by taking trace of the final proton-antiproton density matrix.

For the process $e^{+} e^{-} \rightarrow \Lambda \bar{\Lambda}$ with $\Lambda \rightarrow p \pi^{-}+$c.c. the joint angular distribution is [18]:

$$
\mathcal{W}^{\Lambda \bar{\Lambda}}(\boldsymbol{\xi} ; \boldsymbol{\omega})=\sum_{\mu, \nu=0}^{3} C_{\mu \nu} a_{\mu 0}^{\Lambda} a_{\nu 0}^{\bar{\Lambda}},
$$

where the production reaction is described by the corresponding $C_{\mu \nu}\left(\theta ; \alpha_{\psi}, \Delta \Phi\right)$ matrix, $a_{\mu 0}^{\Lambda} \equiv a_{\mu 0}\left(\theta_{p}, \varphi_{p} ; \alpha_{\Lambda}\right)$ and $a_{\nu 0}^{\bar{\Lambda}} \equiv a_{\nu 0}\left(\theta_{\bar{p}}, \varphi_{\bar{p}} ; \alpha_{\bar{\Lambda}}\right)$ The vector $\boldsymbol{\xi} \equiv\left(\theta, \theta_{p}, \varphi_{p}, \theta_{\bar{p}}, \varphi_{\bar{p}}\right)$ represents a complete set of the kinematic variables describing a single event configuration in the five dimensional phase space. There are four parameters to describe the angular distribution $\boldsymbol{\omega} \equiv\left(\alpha_{\psi}, \Delta \Phi, \alpha_{\Lambda}, \alpha_{\bar{\Lambda}}\right)$.
For the $e^{+} e^{-} \rightarrow \Xi^{-} \bar{\Xi}^{+}$reaction (the formalism for $\Xi^{0} \bar{\Xi}^{0}$ is the same) with the $\Xi^{-} \rightarrow \Lambda \pi^{-}, \Lambda \rightarrow p \pi^{-}+$c.c. decay sequences the joint angular distribution is [18]:

$$
\mathcal{W}^{\Xi \bar{\Xi}}(\boldsymbol{\xi}, \boldsymbol{\omega})=\sum_{\mu, \nu=0}^{3} C_{\mu \nu} \sum_{\mu^{\prime}, \nu^{\prime}=0}^{3} a_{\mu \mu^{\prime}}^{\Xi} a_{\nu \nu^{\prime}}^{\bar{\Xi}} a_{\mu^{\prime} 0}^{\Lambda} a_{\nu^{\prime} 0}^{\bar{\Lambda}},
$$

where $a_{\mu \mu^{\prime}}\left(\theta_{\Lambda}, \varphi_{\Lambda} ; \alpha_{\Xi}, \phi_{\Xi}\right), a_{\nu \nu^{\prime}}^{\bar{\Xi}} \equiv a_{\nu \nu^{\prime}}\left(\theta_{\bar{\Lambda}}, \varphi_{\bar{\Lambda}} ; \alpha_{\bar{\Xi}}, \phi_{\bar{\Xi}}\right)$. For $\Xi(\bar{\Xi})$ all elements of the decay matrix are used and dependence on the $\phi_{\Xi}\left(\phi_{\bar{\Xi}}\right)$ should be included. The joint angular distribution Eq. (5) is a function of nine helicity angles: $\boldsymbol{\xi} \equiv\left(\theta, \theta_{\Lambda}, \varphi_{\Lambda}, \theta_{\bar{\Lambda}}, \varphi_{\bar{\Lambda}}, \theta_{p}, \varphi_{p}, \theta_{\bar{p}}, \varphi_{\bar{p}}\right)$ and depends on eight global parameters: $\omega \equiv\left(\alpha_{\psi}, \Delta \Phi, \alpha_{\Xi}, \phi_{\Xi}, \alpha_{\bar{\Xi}}, \phi_{\bar{\Xi}}, \alpha_{\Lambda}\right.$, $\left.\alpha_{\bar{\Lambda}}\right)$. Since all decays of the sequences are two body with constant c.m. momenta the kinematic weight of states in phase space expressed by the sets of helicity angles $\xi$ is given by the isotropic distributions.

The angular distributions (4) and (5) can be rewritten as:

$$
\sum_{k=1}^{m} g_{k}(\boldsymbol{\omega}) \cdot h_{k}(\boldsymbol{\xi}),
$$

where the functions $g_{k}$ and $h_{k}$ depend only on $\boldsymbol{\omega}$ and $\boldsymbol{\xi}$, respectively. The angular distribution in Eq. (5) requires $m=72$ unique functions $g_{k}(\boldsymbol{\omega})$ of the global parameters, while Eq. (4) only $m=7$. For $\Delta \Phi=0$ the number of such terms reduces to $m=56$ and $m=5$, respectively. The asymptotic case $\alpha_{\psi}=1$ and the (pseudo)scalar charmonium decay still require 20 terms for $\Xi \bar{\Xi}$ while only 2 terms for the $\Lambda \bar{\Lambda}$ final state. This suggests the structure of the $\Xi \bar{\Xi}$ pair joint decay products distribution is rich enough to determine all involved decay parameters separately. For example, in all cases the six pairwise products of the $\alpha_{\Xi}$, $\alpha_{\Lambda}, \alpha_{\bar{\Xi}}$, and $\alpha_{\bar{\Lambda}}$ are present.

Before introducing a rigorous method to analyze the exclusive joint angular distributions we make a comment on the inclusive measurement. If in $e^{+} e^{-} \rightarrow \Xi^{-} \bar{\Xi}^{+}$only $\Xi^{-}$ decay products are measured the corresponding angular distribution is obtained by integrating $\mathcal{W}^{\Xi \overline{\bar{\Xi}}}$ over the $\varphi_{\bar{p}}, \varphi_{\bar{\Lambda}}$, $\cos \theta_{\bar{p}}$, and $\cos \theta_{\bar{\Lambda}}$ variables. The integral is $16 \pi^{2}\left(C_{00} \mathcal{T}_{0}+\right.$ $C_{20} \mathcal{T}_{2}$ ) where $\mathcal{T}_{0}$ and $\mathcal{T}_{2}$ are

$$
\begin{aligned}
\mathcal{T}_{0}= & 1+\alpha_{\Xi} \alpha_{\Lambda} \cos \theta_{\Lambda}, \\
\mathcal{T}_{2}= & \sin \varphi_{\Xi} \sin \theta_{\Xi}\left(\alpha_{\Xi}+\alpha_{\Lambda} \cos \theta_{\Lambda}\right) \\
& +\alpha_{\Lambda} \sin \theta_{\Lambda}\left[\sin \varphi_{\Xi} \cos \theta_{\Xi}\left(\gamma_{\Xi} \cos \varphi_{\Lambda}-\beta_{\Xi} \sin \varphi_{\Lambda}\right)\right. \\
& \left.+\cos \varphi_{\Xi}\left(\beta_{\Xi} \cos \varphi_{\Lambda}+\gamma_{\Xi} \sin \varphi_{\Lambda}\right)\right] .
\end{aligned}
$$

If $\beta_{\psi}=0$ (no polarization) only $\mathcal{T}_{0}$ contributes implying $\alpha_{\Xi}$ and $\alpha_{\Lambda}$ cannot be determined separately as the distribution is given by the product $\alpha_{\Xi} \alpha_{\Lambda}$.

In general the importance of the individual parameters $\omega_{k}$ in the joint angular distributions Eqs. (4) and (5) and 
their correlations are best studied using properties of the corresponding likelihood function. In the ideal case when the response function is diagonal the likelihood function can be written as:

$$
\mathcal{L}(\boldsymbol{\omega})=\prod_{i=1}^{N} \mathcal{P}\left(\boldsymbol{\xi}_{i}, \boldsymbol{\omega}\right) \equiv \prod_{i=1}^{N} \frac{\mathcal{W}\left(\boldsymbol{\xi}_{i}, \boldsymbol{\omega}\right)}{\int \mathcal{W}(\boldsymbol{\xi}, \boldsymbol{\omega}) d \boldsymbol{\xi}},
$$

where $N$ is the number of events in the final selection and $\boldsymbol{\xi}_{i}$ is the full set of kinematic variables describing the $i$ th event. The asymptotic expression of the inverse covariance matrix element between parameters $\omega_{k}$ and $\omega_{l}$ from the vector parameter $\boldsymbol{\omega}$ is given by [7]:

$$
V_{k l}^{-1}=E\left(-\frac{\partial^{2} \ln \mathcal{L}}{\partial \omega_{k} \partial \omega_{l}}\right)
$$

where $E(h)$ denotes the expectation value of a random variable $h(\boldsymbol{\xi})$. Equation (9) can be reduced to:

$$
V_{k l}^{-1}=N \int \frac{1}{\mathcal{P}} \frac{\partial \mathcal{P}}{\partial \omega_{k}} \frac{\partial \mathcal{P}}{\partial \omega_{l}} d \boldsymbol{\xi}
$$

The above integral involves inverse of the angular distribution $\mathcal{W}$ and has to be evaluated numerically. We use the weighted Monte Carlo method to calculate the integrals. The calculated values are then used to construct the matrix, which is inverted to get the covariances for the parameters. If two or more parameters are fully correlated and their values cannot be determined separately the matrix is singular. We report the resulting uncertainties multiplied by $\sqrt{N}$, and call such quantity sensitivity.

TABLE II. Decay parameters of $\Lambda$ and $\Xi$ used in this analysis. They are from the 2019 update of PDG [7] which includes the new $\alpha_{\Lambda}$ value from BESIII [13]. For the charge conjugation decay modes $\alpha_{D}=-\alpha_{\bar{D}}$ and $\phi_{D}=-\phi_{\bar{D}}$.

\begin{tabular}{lrc}
\hline \hline & $\alpha_{D}$ & \multicolumn{1}{c}{$\phi_{D}$} \\
\hline$\Lambda \rightarrow p \pi^{-}$ & $0.750 \pm 0.010$ & $\ldots$ \\
$\Xi^{-} \rightarrow \Lambda \pi^{-}$ & $-0.392 \pm 0.008$ & $-0.037 \pm 0.014$ \\
$\Xi^{0} \rightarrow \Lambda \pi^{0}$ & $-0.347 \pm 0.010$ & $0.37 \pm 0.21$ \\
\hline \hline
\end{tabular}

\section{RESULTS}

We start by verifying the method using the $e^{+} e^{-} \rightarrow$ $J / \psi \rightarrow \Lambda \bar{\Lambda}$ reaction. Here all parameters, including the phase $\Delta \Phi=0.740 \pm 0.010 \pm 0.008$, are known (Table II and Ref. [13]) and we can cross check our estimates of the uncertainties shown in the first row of Table III. To compare with the BESIII statistical uncertainties (in parentheses) we set $N$ to $0.42 \times 10^{6}: \sigma\left(\alpha_{\Lambda}\right)=0.010(0.010), \quad \sigma\left(\alpha_{\psi}\right)=$ $0.005(0.006)$ and $\sigma(\Delta \Phi)=0.012(0.010)$. The agreement is satisfactory since no efficiency variation is included in our calculations. In particular, the $\Lambda$ emission angle is limited to the range $|\cos \theta|<0.85$ in BESIII. Our correlation coefficient between $\alpha_{\Lambda}$ and $\alpha_{\bar{\Lambda}}$ is 0.87 to be compared to 0.82 from the BESIII fit.

To study the angular distribution for the $e^{+} e^{-} \rightarrow \Xi^{-} \bar{\Xi}^{+}$ reaction we fix the decay parameters of the $\Lambda$ and $\Xi^{-}$to the central values listed in Table II. For the production process the main unknown parameter is the phase $\Delta \Phi$ and therefore we use the extreme cases: $\Delta \Phi=0$ and $\pi / 2$. In Table III we report the sensitivities in the $J / \psi \rightarrow \Xi^{-} \bar{\Xi}^{+}$decay. Correlations between parameters are given in Table IV. The results practically do not change between the two $\Delta \Phi$ cases. The results for other decays: $\psi^{\prime} \rightarrow \Xi^{-} \bar{\Xi}^{+}$and $J / \psi, \psi^{\prime} \rightarrow \Xi^{0} \bar{\Xi}^{0}$ are similar. In the table the results for the $e^{+} e^{-} \rightarrow \Xi^{-} \bar{\Xi}^{+}$asymptotic case with $\alpha_{\psi}=1$ and for a scalar charmonium decay to $\Xi \bar{\Xi}$ are also shown. We conclude that contrary to $e^{+} e^{-} \rightarrow \Lambda \bar{\Lambda}$ the polarization in the production process plays practically no role. We find that the weak decay phases $\phi_{\Xi}$ and $\phi_{\bar{\Xi}}$ are not correlated with each other and with any other parameter. Also, the use of parameter input values for $\Xi^{-}$or $\Xi^{0}$ from Table II have only minor effect on the sensitivities.

For $e^{+} e^{-} \rightarrow J / \psi \rightarrow \Xi^{-} \bar{\Xi}^{+}$we also consider single tag measurement and determine correlation coefficient $\rho\left(\alpha_{\Xi}, \alpha_{\Lambda}\right)$ between $\alpha_{\Xi}$ and $\alpha_{\Lambda}$. It is equal to one for $\Delta \Phi=$ 0 and the dependence on $\Delta \Phi$ is well represented by the relation $\rho\left(\alpha_{\Xi}, \alpha_{\Lambda}\right)=(1-p) \cos (\Delta \Phi)+p$, where $p \approx 0.91$. Sensitivity for the product $\alpha_{\Xi} \alpha_{\Lambda}$ is 1.7 , nearly independent on the $\Delta \Phi$ value. The best sensitivity for $\phi_{\Xi}$, with $\Delta \Phi=$ $\pi / 2$ is 12.4 , i.e., at least two times worse than in the exclusive measurement, while for $\Delta \Phi<0.2$ the sensitivity for $\phi_{\Xi}$ can be approximately described by $12.5 \cot (\Delta \Phi)$.

\begin{tabular}{|c|c|c|c|c|c|c|c|c|c|c|c|c|c|}
\hline & $\alpha_{\Xi}$ & $\alpha_{\Lambda}$ & $\phi_{\Xi}$ & $\alpha_{\psi}$ & $\Delta \Phi$ & $\left\langle\alpha_{\Xi}\right\rangle$ & $A_{\Xi}$ & $\left\langle\alpha_{\Lambda}\right\rangle$ & $A_{\Lambda}$ & $\left\langle\alpha_{\Xi} \alpha_{\Lambda}\right\rangle$ & $A_{\Xi \Lambda}$ & $\left\langle\phi_{\Xi}\right\rangle$ & $B_{\Xi}$ \\
\hline$J / \psi \rightarrow \Lambda \bar{\Lambda}$ & $\cdots$ & 6.8 & $\cdots$ & 3.4 & 7.5 & $\cdots$ & $\cdots$ & 1.8 & 8.8 & $\cdots$ & $\cdots$ & $\cdots$ & $\cdots$ \\
\hline$J / \psi \rightarrow \Xi^{-\bar{\Xi}^{+}}(\Delta \Phi=0)$ & 2.0 & 3.1 & 5.8 & 3.5 & 6.0 & 1.4 & 3.7 & 1.7 & 3.5 & 0.78 & 4.0 & 4.1 & 110 \\
\hline$J / \psi \rightarrow \Xi^{-} \bar{\Xi}^{+}(\Delta \Phi=\pi / 2)$ & 1.9 & 2.8 & 5.4 & 3.0 & 13 & 1.4 & 3.5 & 1.6 & 3.1 & 0.76 & 3.9 & 3.8 & 100 \\
\hline$J / \psi \rightarrow \Xi^{0} \bar{\Xi}^{0}(\Delta \Phi=\pi / 2)$ & 2.0 & 3.0 & 5.2 & 2.9 & 15 & 1.4 & 4.0 & 1.5 & 3.4 & 0.77 & 4.4 & 3.7 & 10 \\
\hline$e^{+} e^{-} \rightarrow \Xi^{-} \bar{\Xi}^{+}\left(\alpha_{\psi}=1\right)$ & 1.9 & 2.7 & 5.0 & $\cdots$ & $\cdots$ & 1.3 & 3.4 & 1.4 & 3.1 & 0.76 & 4.0 & 3.5 & 96 \\
\hline$\underline{\eta_{c}, \chi_{c 0} \rightarrow \Xi^{-} \bar{\Xi}^{+}}$ & 1.6 & 2.2 & 3.7 & $\cdots$ & $\cdots$ & 1.1 & 2.9 & 1.0 & 2.6 & 0.72 & 3.9 & 2.6 & 71 \\
\hline
\end{tabular}

TABLE III. Sensitivities (standard errors multiplied by $\sqrt{N}$ ) for the extracted parameters. Errors for the parameters of the charge conjugated decay modes are the same. The input values of the parameters are from Tables I and II. 
TABLE IV. Correlation matrix for the parameters in the $e^{+} e^{-} \rightarrow J / \psi \rightarrow \Xi^{+} \bar{\Xi}^{-}$process. $\Delta \Phi=0$ case (above the diagonal) and $\Delta \Phi=\pi / 2$ case (below the diagonal). Only correlation coefficients with the absolute value greater than 0.01 are shown.

\begin{tabular}{lccccr}
\hline \hline & $\alpha_{\Xi}$ & $\alpha_{\Xi}$ & $\alpha_{\Lambda}$ & $\alpha_{\bar{\Lambda}}$ & $\alpha_{\psi}$ \\
\hline$\alpha_{\Xi}$ & 1 & 0.03 & 0.37 & -0.11 & -0.03 \\
$\alpha_{\Xi}$ & -0.01 & 1 & -0.11 & 0.37 & 0.03 \\
$\alpha_{\Lambda}$ & 0.31 & -0.07 & 1 & 0.43 & -0.12 \\
$\alpha_{\bar{\Lambda}}$ & 0.07 & 0.31 & 0.39 & 1 & 0.12 \\
$\alpha_{\psi}$ & & & -0.04 & 0.04 & 1 \\
\hline \hline
\end{tabular}

An exclusive experiment allows us to determine both the average values and differences of the decay parameters for the charge conjugated modes, which, e.g., for the $\phi_{D}$ parameter are defined as:

$$
\left\langle\phi_{D}\right\rangle \equiv \frac{\phi_{D}-\phi_{\bar{D}}}{2} \quad \text { and } \quad \Delta \phi_{D} \equiv \frac{\phi_{D}+\phi_{\bar{D}}}{2} .
$$

The $C P$ asymmetry $A_{D}$ is defined as:

$$
A_{D} \equiv \frac{\alpha_{D}+\alpha_{\bar{D}}}{\alpha_{D}-\alpha_{\bar{D}}}
$$

and $B_{D}$ as:

$$
B_{D} \equiv \frac{\beta_{D}+\beta_{\bar{D}}}{\beta_{D}-\beta_{\bar{D}}} \approx-\frac{\left\langle\alpha_{D}\right\rangle \Delta \alpha_{D}}{1-\left\langle\alpha_{D}\right\rangle^{2}}+\frac{\Delta \phi_{D}}{\tan \left\langle\phi_{D}\right\rangle},
$$

where the approximate form includes only linear terms in $\Delta \alpha_{D}$ and $\Delta \phi_{D}$. Since the phase $\left\langle\phi_{\Xi}\right\rangle$ is small, the last term in Eq. (13) dominates and $B_{\Xi} \approx \Delta \phi_{\Xi} /\left\langle\phi_{\Xi}\right\rangle$. The sensitivities for the $A_{\Xi}, A_{\Lambda}, A_{\Xi \Lambda}$, and $B_{\Xi}$ asymmetries are given in Table III. The sensitivity for $A_{\Lambda}$ is 2.5 times better in $J / \psi \rightarrow \Xi^{-} \bar{\Xi}^{+}$ than in $J / \psi \rightarrow \Lambda \bar{\Lambda}$. The statistical uncertainty for the $A_{\Xi \Lambda}$ asymmetry from the dedicated HyperCP experiment could be surpassed at STCF in a run at the $J / \psi$ c.m. energy with more than $10^{12}$ events. The SM predictions for the $A_{\Xi}$ and $A_{\Lambda}$ asymmetries are $-3 \times 10^{-5} \leq A_{\Lambda} \leq 4 \times 10^{-5}$ and $-2 \times$ $10^{-5} \leq A_{\Xi} \leq 1 \times 10^{-5}[9]$.

A prerequisite for a complementary $C P$ test using $B_{\Xi}$ asymmetry, advocated in Ref. [6] as the most sensitive probe, is $\left\langle\phi_{\Xi}\right\rangle \neq 0$. Assuming $\left\langle\phi_{\Xi}\right\rangle=0.037$, according to the Table II value for $\Xi^{-}$, the five sigma significance requires $3.1 \times 10^{5}$ exclusive $\Xi^{-} \bar{\Xi}^{+}$events. To reach the statistical uncertainty of 0.011 , as in the HyperCP experiment [22] requires $1.4 \times 10^{5} \mathrm{~J} / \psi \rightarrow \Xi^{-} \bar{\Xi}^{+}$events, while the single cascade HyperCP result is based on $114 \times 10^{6}$ events. The present PDG precision of $\phi_{\Xi^{0}}$ can be achieved with just $3 \times 10^{2} \Xi^{0} \bar{\Xi}^{0}$ events. The SM estimate for $B_{\Xi}$ is $8.4 \times 10^{-4}$, an order of magnitude larger compared to the $A$ asymmetries $[6,23]$, while the sensitivities for $B_{\Xi}$ in Table III are 20-30 times worse. However, it should be stressed that the SM predictions for all asymmetries need to be updated in view of the recent and forthcoming BESIII results on hyperon decay parameters. Our analysis shows that a wide range of $C P$ precision tests can be conducted in a single measurement. Thus, the spin entangled cascadeanticascade system is a promising probe for testing fundamental symmetries in the strange baryon sector.

\section{ACKNOWLEDGMENTS}

A. K.'s work was supported in part by National Natural Science Foundation of China (NSFC) under Contract No. 11935018.
[1] J. H. Christenson, J. W. Cronin, V. L. Fitch, and R. Turlay, Phys. Rev. Lett. 13, 138 (1964).

[2] B. Aubert et al. (BABAR Collaboration), Phys. Rev. Lett. 87, 091801 (2001).

[3] K. Abe et al. (Belle Collaboration), Phys. Rev. Lett. 87, 091802 (2001).

[4] R. Aaij et al. (LHCb Collaboration), Phys. Rev. Lett. 122, 211803 (2019).

[5] R. Aaij et al. (LHCb Collaboration), Nat. Phys. 13, 391 (2017).

[6] J. F. Donoghue and S. Pakvasa, Phys. Rev. Lett. 55, 162 (1985).

[7] M. Tanabashi et al. (Particle Data Group), Phys. Rev. D 98, 030001 (2018), and 2019 update.

[8] T. Holmstrom et al. (HyperCP Collaboration), Phys. Rev. Lett. 93, 262001 (2004).
[9] J. Tandean and G. Valencia, Phys. Rev. D 67, 056001 (2003).

[10] C. Materniak (HyperCP Collaboration), Nucl. Phys. B, Proc. Suppl. 187, 208 (2009).

[11] D. M. Asner et al., Int. J. Mod. Phys. A 24, S1 (2009).

[12] C.-Z. Yuan and S. L. Olsen, Nat. Rev. Phys. 1, 480 (2019).

[13] M. Ablikim et al. (BESIII Collaboration), Nat. Phys. 15, 631 (2019).

[14] G. Fäldt and A. Kupść, Phys. Lett. B 772, 16 (2017).

[15] M. Ablikim et al. (BESIII Collaboration), Phys. Rev. D 95, 052003 (2017).

[16] M. Ablikim et al. (BESIII Collaboration), Phys. Lett. B 770, 217 (2017).

[17] M. Ablikim et al. (BESIII Collaboration), Phys. Rev. D 93, 072003 (2016).

[18] E. Perotti, G. Fäldt, A. Kupsc, S. Leupold, and J. J. Song, Phys. Rev. D 99, 056008 (2019). 
[19] E. B. Levichev, A. N. Skrinsky, G. M. Tumaikin, and Y. M. Shatunov, Phys. Usp. 61, 405 (2018).

[20] Q. Luo and D. Xu, in Proceedings, 9th International Particle Accelerator Conference (IPAC 2018): Vancouver, BC Canada (2018), p. MOPML013, https://doi.org/ 10.18429/JACoW-IPAC2018-MOPML013.
[21] S. J. Brodsky and G. P. Lepage, Phys. Rev. D 24, 2848 (1981).

[22] M. Huang et al. (HyperCP Collaboration), Phys. Rev. Lett. 93, 011802 (2004).

[23] J. F. Donoghue, X.-G. He, and S. Pakvasa, Phys. Rev. D 34, 833 (1986). 